\title{
Current Overview on Hypercoagulability in COVID-19
}

\author{
Namrata Singhania ${ }^{1}$ - Saurabh Bansal ${ }^{2}$. Divya P. Nimmatoori ${ }^{3}$. Abutaleb A. Ejaz ${ }^{4}$ Peter A. McCullough ${ }^{5,6,7,8}$. \\ Girish Singhania ${ }^{9,10}$
}

Published online: 4 August 2020

(c) Springer Nature Switzerland AG 2020

\begin{abstract}
The coronavirus disease 2019 (COVID-19) pandemic, caused by severe acute respiratory syndrome coronavirus 2 (SARS$\mathrm{CoV}-2$ ), has brought many unique pathologies, such as coagulopathy, prompting a desperate need for effective management. COVID-19-associated coagulopathy (CAC) can cause various thromboembolic complications, especially in critically ill patients. The pathogenesis is likely due to endothelial injury, immobilization, and an increase in circulating prothrombotic factors. Data on treatment are limited, although prophylactic anticoagulation is advised in all hospitalized patients. Herein, we have comprehensively reviewed the current literature available on CAC and highlight the pathogenesis, clinical features, and management of CAC.
\end{abstract}

Namrata Singhania

namrat09@gmail.com

1 Department of Hospital Medicine, Mount Carmel East Hospital, 6001 E Broad Street, Columbus, OH 43213, USA

2 Department of Internal Medicine, University of Illinois at Peoria, Peoria, IL 61637, USA

3 Department of Internal Medicine, Greenfield Health, Portland, OR, USA

4 Department of Internal Medicine, University of Florida, Gainesville, FL, USA

5 Department of Cardiovascular Medicine, Baylor Heart and Vascular Institute, Dallas, TX, USA

6 Department of Internal Medicine, Texas A\&M College of Medicine Health Science Center, Dallas, TX, USA

7 Department of Internal Medicine, Baylor University Medical Center, Dallas, TX, USA

8 Department of Cardiovascular Medicine, Baylor Heart and Vascular Hospital, Dallas, TX, USA

9 Department of Hospital Medicine, CHI St Vincent Infirmary, Little Rock, AR, USA

10 Department of Nephrology and Hypertension, University of Utah, Salt Lake City, UT, USA

\section{Key Messages}

Venous thromboembolism (VTE) is common in COVID19 patients, especially those in the intensive care unit.

Prophylactic anticoagulation is recommended in all patients with COVID-19 unless contraindicated.

\section{Introduction}

A novel coronavirus was first identified in late 2019 in Wuhan, China, and rapidly spread throughout the world, causing a pandemic [1]. The virus was identified as severe acute respiratory syndrome coronavirus 2 (SARS-CoV-2), and the World Health Organization designated the disease as coronavirus disease 2019 (COVID-19). The most common symptoms are respiratory, but gastrointestinal, neurologi$\mathrm{cal}$, and other atypical symptoms can also be seen, although these symptoms are rare [2]. Recent studies showed several coagulation abnormalities in patients with COVID-19, raising questions about appropriate management to prevent or treat thrombosis; this state has been termed COVID-19-associated coagulopathy (CAC) [3]. The International Society on Thrombosis and Hemostasis (ISTH), the American Society of Hematology (ASH), and the American College of Cardiology (ACC) have posted interim guidance on this topic. 
Herein, we provide a clinical overview on the pathogenesis, clinical features, and management of hypercoagulability in individuals with COVID-19.

\section{Pathogenesis}

The pathogenesis of hypercoagulability in COVID-19 is illdefined. Figure 1 summarizes the proposed pathogenesis of hypercoagulability in COVID-19; all three components of Virchow's triad appear to be involved, including endothelial injury, stasis, and hypercoagulable state. Endothelial injury is evident from the direct invasion of endothelial cells by SARS-CoV-2 [4]; endothelial cells have a high number of angiotensin-converting enzyme 2 (ACE-2) receptors. SARS-CoV-2 enters the cell through the ACE-2 receptor [4]. In the study by Varga et al., viral elements were found inside the endothelial cells, suggesting direct invasion [4]. Increased angiogenesis was also seen in these patients [5]. Increased cytokines are released, such as interleukin (IL)-6, and various acute-phase reactants in COVID-19 can lead to endothelial injury [6]. Reports also suggest activation of alternate and lectin complement pathways $\left(\mathrm{C}_{5 \mathrm{~b}-9}\right.$ [membrane attack complex], $\mathrm{C} 4 \mathrm{~d}$, and mannose-binding proteinassociated serine protease 2 [MASP2]), leading to further endothelial cell injury [7]. The use of intravascular catheters can cause direct endothelial cell injury. Stasis is due to immobilization in all hospitalized patients, especially those who are critically ill. A hypercoagulable state is seen due to several coagulation abnormalities from elevated circulating prothrombotic factors such as elevated von Willebrand factor (vWF), factor VIII, D-dimer, fibrinogen, neutrophil extracellular traps, prothrombotic microparticles, and anionic phospholipids [8]. Elevated levels of D-dimer have been observed to correlate with illness severity and 28-day mortality [9]. Fibrinogen levels were also significantly $(p=0.003)$ associated with IL-6 levels at baseline, according to a logarithmic regression [8].

Upon autopsy, most patients showed macro- and microvascular thrombosis [10]. Gross examination of the lungs

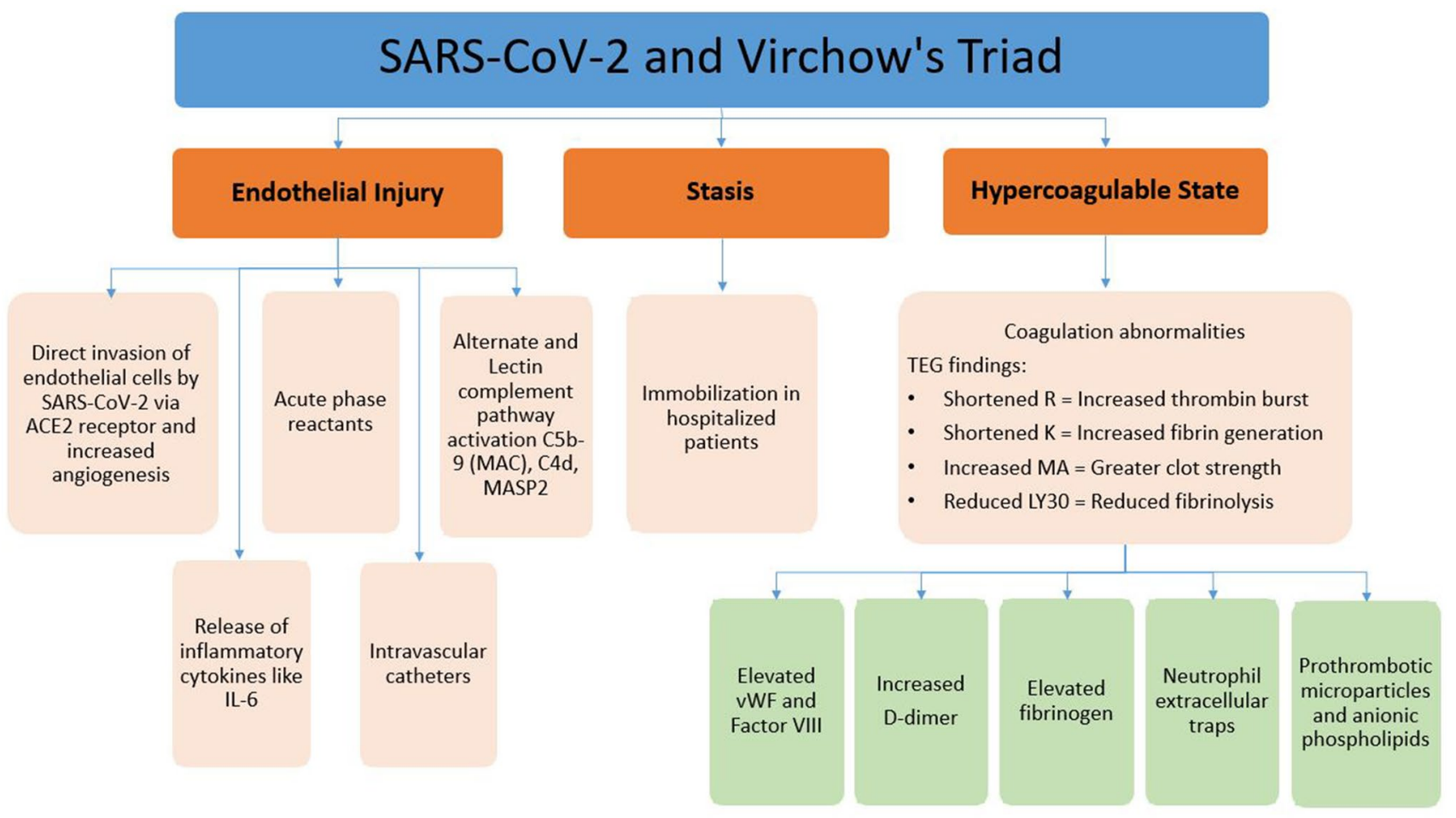

Fig. 1 Pathogenesis of coagulopathy in COVID-19. Endothelial injury (endotheliitis) is caused by direct invasion of endothelial cells by the SARS-CoV-2 virus via ACE-2 receptors, release of inflammatory cytokines such as IL-6, acute-phase reactants, complement activation, and direct injury from intravascular catheters. Stasis is due to immobilization in all hospitalized patients. The hypercoagulable state is due to elevated circulating prothrombotic factors such as elevated vWF activity, factor VIII, D-dimer, fibrinogen, neutrophil extracellular traps, prothrombotic microparticles, and anionic phospholipids. TEG findings showed shortened R (increased early thrombin burst), shortened K (increased fibrin generation), increased MA (greater clot strength), and reduced LY30 (reduced fibrinolysis). $A C E-2$ angiotensin-converting enzyme 2, $C 4 d$ complement $4 \mathrm{~d}, C 5 b-9$ complement 5b-9, COVID-19 coronavirus disease 2019, IL interleukin, $K$ clot formation time, $L Y 30$ clot lysis at $30 \mathrm{~min}, M A$ maximum amplitude, $M A C$ membrane attack complex, MASP2 mannose-binding proteinassociated serine protease $2, R$ reaction time, SARS-CoV-2 severe acute respiratory syndrome coronavirus $2, T E G$ thromboelastography, $v W F$ von Willebrand factor 
showed small and firm thrombi in peripheral parenchyma [5]. The pathological hallmark of COVID-19 is diffuse, small-vessel platelet-fibrin thrombi and intravascular megakaryocytes in all major organs, including the heart, lungs, kidneys, liver, and mesenteric fat [10]. Microscopic findings demonstrated pauci-inflammatory capillary injury, capillary congestion with luminal fibrin deposition, and angiogenesis $[5,10]$. The density of intussusceptive angiogenic features (mean \pm standard error [SE]) $60.7 \pm 11.8$ features per field) was significantly higher in the lungs from patients with COVID-19 than from patients with influenza $(22.5 \pm 6.9)$ or from uninfected controls $(2.1 \pm 0.6)[p<0.001$ for both comparisons] [5]. The degree of intussusceptive angiogenesis correlated significantly with the duration of hospitalization $(p<0.001)$. Endotheliitis is visible by electron microscopy in the endothelium of the heart, lung, small bowel, and kidneys [4]. Autopsy of the kidneys showed diffuse tubular injury, interstitial edema, and fibrin thrombi in the glomerular capillaries [11].

Routine laboratory testing was performed in 24 critically ill COVID-19 patients and identified several abnormalities, including normal or slightly prolonged prothrombin time (PT) and activated partial thromboplastin time (aPTT), normal or increased platelet count, and increased D-dimer and fibrinogen levels [12]. Further testing also showed increased factor VIII activity and vWF antigens. Thromboelastography (TEG) showed a shortened reaction time $(R)$, consistent with an increased early thrombin burst, in 50\% of patients; shortened clot formation time $(K)$, consistent with increased fibrin generation, in $83 \%$ of patients; increased maximum amplitude (MA), consistent with greater clot strength, in 83\% of patients; and reduced clot lysis at $30 \mathrm{~min}$ (LY30), consistent with reduced fibrinolysis, in $100 \%$ of patients [12]. Similarly, in another viscoelastic test, increased platelet and fibrinogen contribution to clot strength was found [8]. In patients with severe disease and a positive lupus anticoagulant test, thrombocytopenia and marked prolongation of PT and aPTT can be seen [13].

Although disseminated intravascular coagulation (DIC) has been rarely reported in severely ill patients with COVID-19, there are several features that distinguish CAC from DIC (Table 1). The major clinical finding in CAC is thrombosis, whereas in acute decompensated DIC, bleeding is the predominant feature. Pathological bleeding is not a commonly observed feature of COVID-19. Furthermore, COVID-19 has a few findings similar to DIC, including a marked increase in D-dimer levels and, in some cases, mild thrombocytopenia [14]. However, other coagulation parameters such as fibrinogen and factor VIII activity are high in CAC, while acute decompensated DIC is associated with low fibrinogen, which suggests that consumption of clotting factors is less likely in CAC. Regardless of the above similarities or differences, the basic principle of management in both CAC and DIC is treatment of the underlying condition.

\section{Clinical Features}

\subsection{Venous Thromboembolism}

Venous thromboembolism (pulmonary embolism [PE] and/ or deep vein thrombosis [DVT]) is common in patients with COVID-19, even when prophylactic anticoagulation is used, and can be seen in up to two-thirds of patients in the intensive care unit (ICU). A search of the PubMed database using the key words 'hypercoagulability' and 'COVID-19' found 11 studies with a patient population of 10 or more. Details of the studies, including type, country, number of patients enrolled, mean age, sex, comorbidities, use of anticoagulation, incidence of thromboembolism, and other relevant laboratory findings are listed in Table 2 . Two of 11 studies were autopsy studies. Older age, male sex, obesity, smoking

Table 1 Distinguishing features between COVID-19-associated coagulopathy and acute disseminated intravascular coagulation

\begin{tabular}{lll}
\hline & COVID-19-associated coagulopathy & Acute disseminated intravascular coagulation \\
\hline Major clinical finding & Thrombosis & Bleeding \\
PT & Normal/increased & Increased \\
APTT & Normal/increased & Increased \\
Platelet counts & Normal/increased/decreased & Decreased \\
Fibrinogen & Increased & Decreased \\
D-dimer & Increased & Increased \\
VWF and factor VIII activity & Increased & Increased \\
Antithrombin & Increased & Decreased \\
Anticardiolipin Antibody & Positive & Normal \\
Protein C & Increased & Decreased \\
\hline
\end{tabular}

aPTT activated partial thromboplastin time, COVID-19 coronavirus disease 2019, PT prothrombin time, $v W F$ von Willebrand factor 
and other chronic medical comorbidities, especially cardiovascular disease, hypertension, chronic bronchitis, active cancer, and diabetes mellitus were associated with a higher risk of thromboembolism.

In a post-mortem examination of 21 patients with COVID-19, alveolar and glomerular microthrombi were seen in $45 \%$ and $16.7 \%$ of individuals, respectively, while in another post-mortem study of 12 patients, DVT was seen in $58 \%$ of patients with $33 \%$ mortality due to a massive PE [11, 15]. Extremely high values of D-dimer $(>20,000 \mathrm{ng} / \mathrm{mL}$; normal value $<500 \mathrm{ng} / \mathrm{mL}$ ) were found in $25 \%$ of patients. In a series of 184 patients (all patients receiving prophylactic anticoagulation) with severe COVID-19 and in the ICU, VTE was reported in 27\% [16]; the study was associated with a $13 \%$ mortality rate. Age (adjusted hazard ratio [aHR] 1.05/year, 95\% confidence interval [CI] 17-37\%) and coagulopathy (prolongation of PT $>3 \mathrm{~s}$ or aPTT $>5 \mathrm{~s}$; aHR $4.1,95 \%$ CI 1.9-9.1) were found to be independent predictors of thrombosis. In another series of 150 ICU patients, 64 patients had clinically relevant thrombotic complications (mostly PE) [17]. Clotting of the extracorporeal circuit was seen in 28 of 29 patients receiving continuous renal replacement therapy (RRT), and 2 of 12 patients undergoing extracorporeal membrane oxygenation (ECMO). All patients received anticoagulation (prophylactic dose, $70 \%$; therapeutic dose, $30 \%$ ). That study also compared patients with COVID-19-associated acute respiratory distress syndrome (ARDS) with a matched cohort of non-COVID-19 ARDS patients and found the rate of PE was higher in COVID19 patients [ $11.7 \%$ vs. $2.1 \%$; odds ratio (OR) $6.2,95 \%$ CI 1.6-23.4; $p<0.008$ ]. More than $95 \%$ of patients had elevated D-dimer and fibrinogen levels, with a considerable increase in vWF activity, vWF antigen, and factor VIII. The study also reported a positive lupus anticoagulant test in $87.7 \%$ of patients.

Similarly, a series of 107 ICU patients reported PE in $20.6 \%$. By comparison, in two matched cohorts (one from the same time interval in the previous year and one from concurrent patients with influenza rather than COVID-19) $6.1 \%$ [absolute risk (AR) 14.4\%, 95\% CI 6.1-22.8\%] and 7.5\% (AR $13.1 \%$, 95\% CI 1.9-24.3\%) of patients, respectively, reported PE [18]. The study also showed elevated D-dimer, plasma factor VIII and vWF antigen levels associated with higher PE risk. In one study of 74 ICU patients, VTE was associated with higher mortality (aHR 2.4, 95\% CI 1.02-5.5 [19]. The cumulative incidence of VTE was higher in the ICU than on the wards. Another study that performed screening leg ultrasounds in 26 patients with COVID-19 in the ICU who were all receiving either prophylactic-dose anticoagulation $(31 \%)$ or therapeutic-dose anticoagulation $(69 \%)$, found VTE in 18 (69\%) patients, with a $12 \%$ mortality rate [20]. The majority of the above data were from ICU patients, however the data are limited in non-ICU patients.
Two studies (314 and 71 non-ICU patients, respectively) found an incidence of VTE in the range of 6.4-21\%, in spite of the majority of patients receiving anticoagulation [21, 22].

\subsection{Arterial Thromboembolism}

Although VTE is more commonly seen with COVID19 , there have been a few cases of arterial events such as ischemic stroke and acute limb ischemia. In one report, five COVID-19 patients younger than 50 years of age were identified with acute ischemic stroke due to large vessel occlusion during a 2-week period, compared with an average of 0.73 patients every 2 weeks over the previous 12 months [23]. The cumulative incidence rate of acute ischemic stroke was found to be between 2 and $3.7 \%$ in ICU patients $[16,17]$ and approximately $2 \%$ in non-ICU patients [21].

A report evaluated data from 20 patients (90\% male; mean age 75 years) with COVID-19 who developed acute limb ischemia at a single institution over a 3-month period and found that the incidence of acute limb ischemia was significantly higher compared with the previous year $(16.3 \%$ in 2020 vs. $1.8 \%$ in 2019 ; $p<0.001$ ) [24]. Surgical revascularization was performed in 17 patients, of whom $12(71 \%)$ were successful. Re-intervention was not required in those who received postoperative heparin. The study was associated with a $40 \%$ mortality rate, which was significantly higher in older patients $(81 \pm 10$ years vs. $71 \pm 5$ years; $p=0.008)$. The use of postoperative heparin infusion was significantly associated with improvement in survival ( $0 \%$ vs. $57.1 \%$; $p=0.42$ ). Another series reported on two young and healthy patients (37 and 53 years of age, respectively) [25]. Both patients had very high D-dimer levels and were receiving prophylactic-dose anticoagulation at the time of the event. There are a few reports on ST elevation myocardial infarction (STEMI) and mesenteric ischemia [17, 26].

\section{Clinical Evaluation}

All patients admitted to the hospital with COVID-19 should get a complete blood count (CBC), and PT, aPTT, fibrinogen, and D-dimer levels should be checked. D-dimer levels have shown to be directly proportional to the severity of the disease. High D-dimer and fibrinogen levels, normal or mildly prolonged PT and aPTT, mild thrombocytopenia or thrombocytosis, or normal platelet count are typically seen. The values of these typical tests were also found to correlate with 28-day mortality based on multivariate analysis of 447 patients in a retrospective study [9]. PT (OR 1.107, 95\% CI 1.008-1.215) and D-dimer (OR 1.058, 95\% CI 1.028-1.090) levels correlated positively, while platelet count (OR 0.996, 95\% CI 0.993-0.998) correlated negatively, with 28-day 


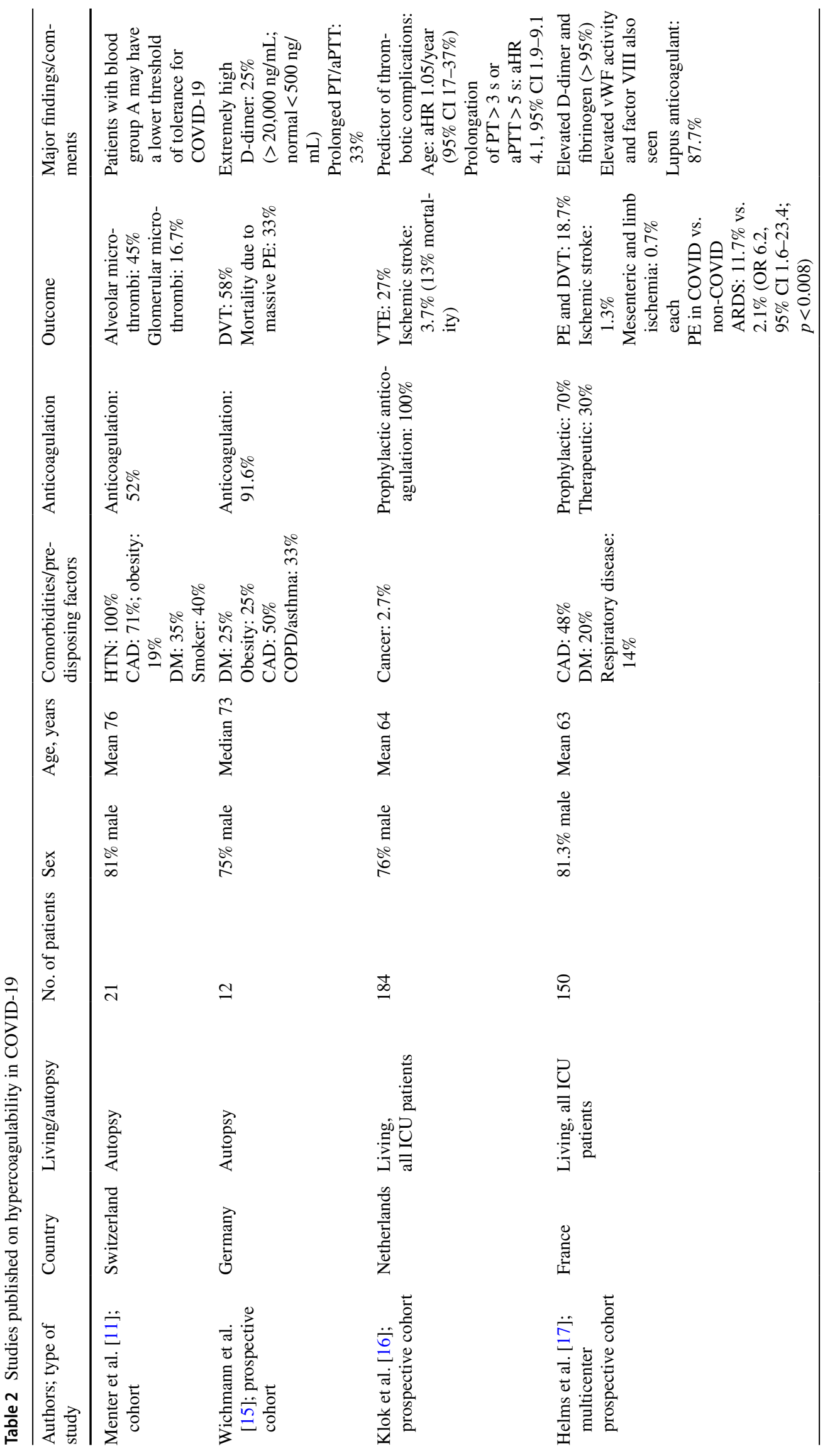




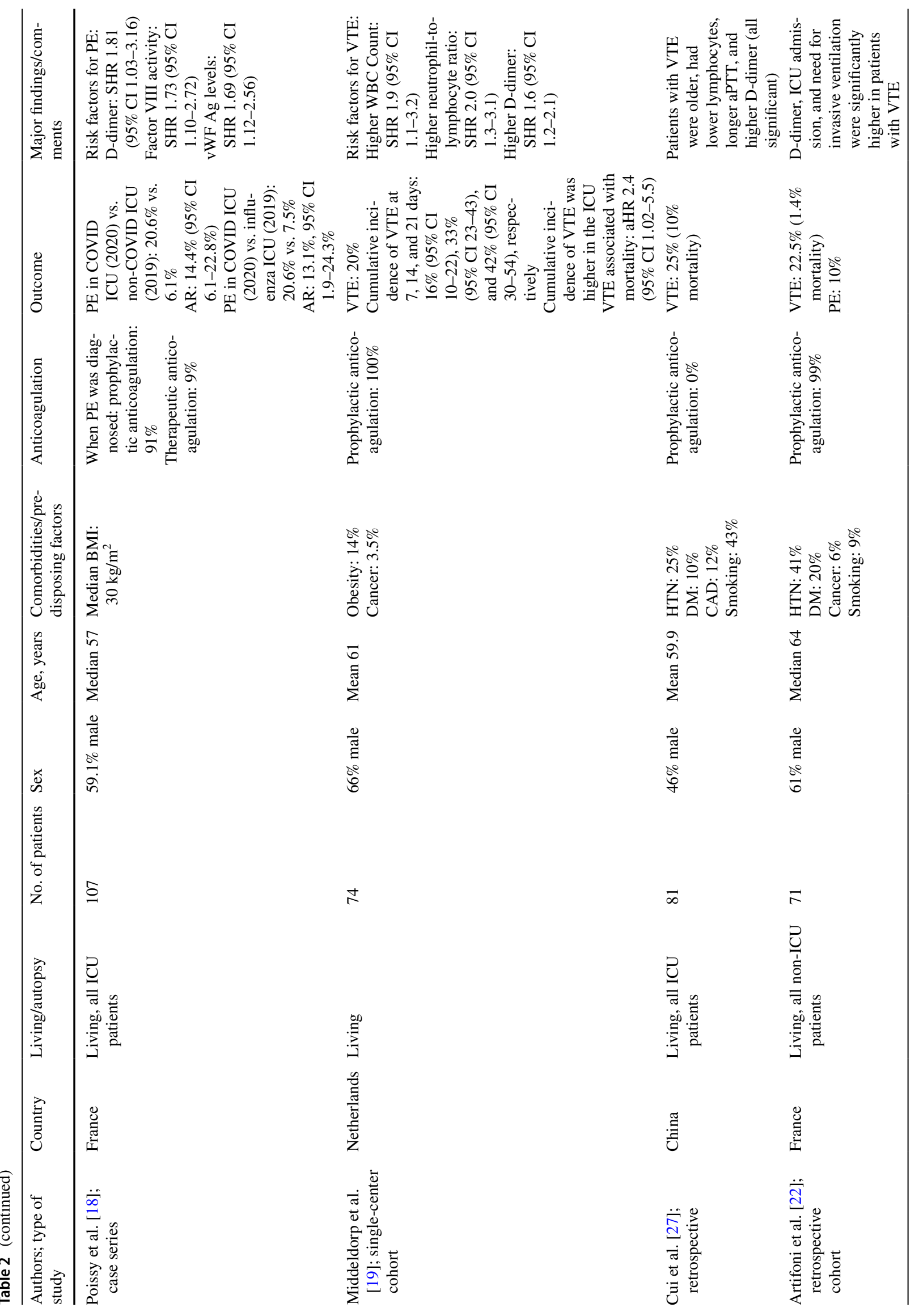




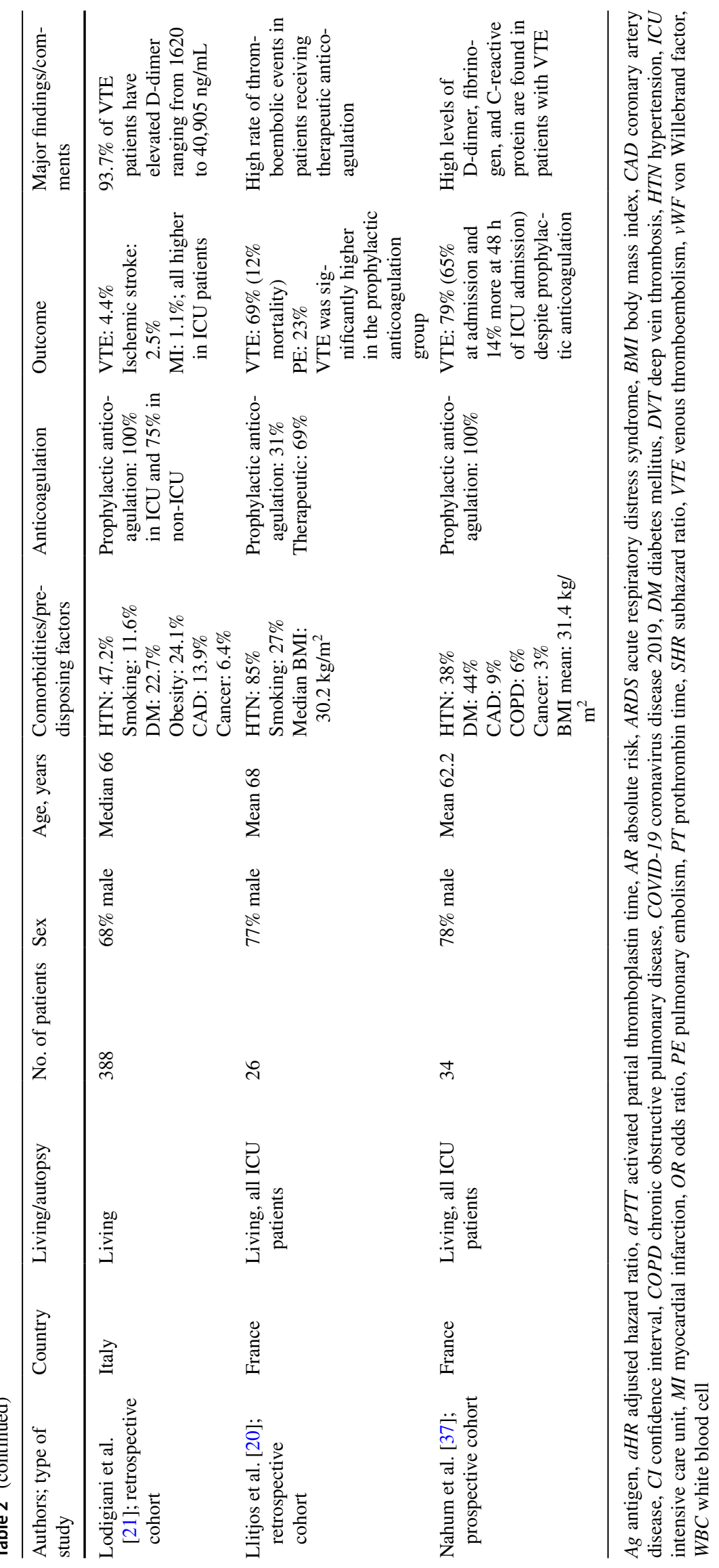


mortality [9]. Few studies have evaluated D-dimer levels at various cut-off values to predict VTE in patients with COVID-19 [27]. Cui et al. showed that if a cut-off value of $1.5 \mu \mathrm{g} / \mathrm{mL}$ was used to predict VTE, the sensitivity was $85.0 \%$, the specificity was $88.5 \%$, and the negative predictive value was $94.7 \%$ [27]. According to ASH, a normal D-dimer may be enough to rule out PE in most stable COVID-19 patients with low to moderate pretest probability, as calculated using the Wells score [3].

Although Yao et al. did not specifically study the correlation between various D-dimer levels and VTE, they showed that D-dimer at a cut-off value of $0.5 \mu \mathrm{g} / \mathrm{mL}$ was present in more than $70 \%$ of patients with COVID-19, and an increase in D-dimer levels correlated significantly with disease severity [28]. As many COVID-19 patients may present with high levels of D-dimer due to inflammation, it is difficult to rule out VTE based on D-dimer alone in patients with high pretest probability [29]. Additional testing, including ultrasonography of the extremities and computed tomography angiography (CTA) of the chest are recommended and should be used readily depending on clinical presentation, due to the high risk of VTE associated with this disease. A ventilation-perfusion (VQ) scan is recommended in patients with contraindications to CTA, such as those with poor renal function. An echocardiogram may be considered in some patients, especially those in whom CTA cannot be performed and in whom a VQ scan has limited value due bilateral infiltrates. An echocardiogram may show right heart strain and a dilated/hypokinetic right ventricle. Additional work-up is recommended in those patients with unexplained hypotension, tachycardia, worsening respiratory status, or other risk factors for thrombosis. Patients with atypical findings such as severe thrombocytopenia and/or markedly prolonged PT/ aPTT require further investigation. If thrombotic microangiopathy is suspected, serum lactate dehydrogenase, haptoglobin, ADAMTS13 activity, and peripheral blood smear are recommended. Antiphospholipid antibody testing is not routinely recommended as it can be transiently present in viral infections.

\section{Management}

The management of hypercoagulability in COVID-19 can be challenging due to limited data. Although all hospitalized patients are at higher risk for thrombosis, ICU patients are at the highest risk (incidence $1.1 \%$ in non-ICU patients vs. $79 \%$ in ICU patients) [3]. It is recommended to start prophylactic-dose anticoagulation with low molecular weight heparin (LMWH) in all acutely ill hospitalized patients unless contraindicated [30]. Many experts suggest initiating intermediate-dose (LMWH 30-40 mg subcutaneously twice daily) or full-dose anticoagulation in critically ill individuals with COVID-19 due to the high percentage of patients with VTE despite receiving prophylactic anticoagulation [16, 31-33]. Due to the lack of high-quality studies favoring such interventions, and the risks of bleeding associated with antithrombotic therapies, some medical societies have deferred from adopting these recommendations in universal guidelines [30]. In addition, data are lacking comparing different doses of anticoagulation (prophylactic or therapeutic) in patients with COVID-19. Most institutions have made their own criteria to manage coagulopathy in these patients. It is advised to balance the risks of thrombosis with bleeding while making decisions regarding antithrombotic therapy. The ISTH has also issued interim guidelines to manage coagulopathy in COVID-19 [34].

In a pre-proof retrospective study of 2773 patients hospitalized with COVID-19, patients who received anticoagulation (786 patients, $21 \%$ ) had an in-hospital mortality rate of $22.5 \%$ and a median survival of 21 days, compared with $22.8 \%$ and a median survival of 14 days in those who did not receive anticoagulation [35]. A significantly higher number of patients required mechanical ventilation in those who did not receive anticoagulation compared with those who did ( $29.8 \%$ vs. $8.1 \%, p<0.001)$. The in-hospital mortality rate was $29.1 \%$ and a median survival of 21 days in a subgroup of intubated patients who received anticoagulation compared with a mortality rate of $62.7 \%$ and a median survival of 9 days in those who were intubated but did not receive anticoagulation. In a multivariate proportional hazard model, a longer duration of anticoagulation was associated with a reduced risk of mortality (aHR 0.86/day, 95\% CI 0.82-0.89; $p<0.001)$. Of a total of 62 patients who received anticoagulation, 24 had bleeding events (not statistically significant); of those 24 patients, 15 had bleeding events after starting anticoagulation. The above study had multiple limitations, such as its retrospective observational nature, unknown reasons for not starting prophylactic anticoagulation in $79 \%$ of patients, unobserved confounding factors, and a lack of metrics to classify patients based on severity. Another retrospective study of 449 patients (99 patients received anticoagulation for $>7$ days) with severe COVID-19 (defined as a respiratory rate of $\geq 30$ breaths/min, arterial oxygen saturation $\leq 93 \%$ at rest, and $\mathrm{PaO}_{2} / \mathrm{FiO}_{2} \leq 300 \mathrm{mmHg}$ ), 28-day mortality benefit was found in patients who received heparin and had either a high sepsis-induced coagulopathy score (40\% with heparin vs. $64.2 \%$ without; $p=0.029$ ) or a high D-dimer of more than sixfold the upper limit of normal (32.8\% vs. $52.4 \% ; p=0.017$ ) [9]. This mortality benefit was not significant in the cohort as a whole.

The recommended prophylactic dose of LMWH for patients with creatinine clearance $(\mathrm{CrCl})>30 \mathrm{~mL} / \mathrm{min}$ is $40 \mathrm{mg}$ once daily, and $30 \mathrm{mg}$ once daily for $\mathrm{CrCl} 15-30 \mathrm{~mL} /$ min. For patients with a body mass index $>40 \mathrm{~kg} / \mathrm{m}^{2}$, it is recommended to increase the dose by $30 \%$. Unfractionated 
heparin should be considered in patients with $\mathrm{CrCl}<15 \mathrm{~mL} /$ min or receiving RRT, or in those where an invasive procedure is anticipated, such as a cesarean section. Other medications, such as dalteparin (5000 units once daily), nadroparin (3800-5700 units once daily), and tinzaparin (4500 units once daily), are alternatives in other parts of the world. Therapeutic-dose (full-dose) anticoagulation (e.g. LMWH $1 \mathrm{mg} /$ kg every $12 \mathrm{~h}$ ) is appropriate in patients with documented VTE, similar to those individuals without COVID-19, and in a few cases of suspected VTE where confirmatory testing is not feasible and the patient developed unexplained respiratory failure with stable chest radiograph, along with elevated D-dimer and/or fibrinogen levels. More than $70 \%$ of the studies summarized in Table 2 showed D-dimer levels being positively correlated with risk for VTE or illness severity. D-dimer levels were in the range of $1600-40,000 \mathrm{ng} / \mathrm{mL}$ (normal range $<500 \mathrm{ng} / \mathrm{mL}$ ) [21]. Full-dose anticoagulation is appropriate for individuals with recurrent clotting of intravascular access devices (arterial lines, central venous catheters) or extracorporeal circuits, such as continuous RRT or ECMO, despite prophylactic-dose anticoagulation. Alternative therapy should be considered in patients with contraindications for heparin, such as heparin-induced thrombocytopenia or active or recent bleeding. Tissue plasminogen activator can be used in cases of arterial thrombosis, such as acute limb ischemia, acute STEMI, acute ischemic stroke, and massive bilateral PE associated with hemodynamic instability. Surgical consultation is recommended in patients with acute mesenteric ischemia. In patients with severe acute kidney injury, initiating RRT is recommended. Patients with COVID-19 and documented VTE need at least 3 months of anticoagulation post-discharge from the hospital, with appropriate follow-up. A few patients who are at high risk, such as those with immobilization due to recent surgery and trauma, may need post-discharge prophylaxis even without documented VTE. Rivaroxaban $10 \mathrm{mg}$ daily for 31-39 days is considered a reasonable alternative as outpatient prophylaxis in such high-risk patients [36].

\section{Conclusions}

VTE is common is critically ill patients with COVID-19. Work-up includes routine testing with CBC, PT, aPTT, fibrinogen, and D-dimer. These tests also correlate with disease severity and mortality. It is recommended to start at least prophylactic-dose anticoagulation in all patients with COVID-19 admitted to the hospital, especially those in the ICU unless contraindicated. If VTE is suspected, confirmatory testing should be obtained to justify full-dose anticoagulation. Some experts support intermediate- or full-dose anticoagulation, even in individuals with COVID-19 who do not have documented VTE, but data on appropriate dosing are limited. In cases of suspected VTE where standard confirmatory testing cannot be obtained, full-dose anticoagulation can be considered in patients with unexplained respiratory failure with stable chest radiograph, along with elevated D-dimer and/or fibrinogen levels. Close monitoring for clinical signs of thrombosis or bleeding is recommended in all patients. Physicians are advised to use their clinical judgment to balance the risks and benefits associated with anticoagulation. Several therapies for COVID-19 are under investigation, some of which may impact thrombotic risk. Participation in clinical trials is encouraged to improve our understanding of pathogenesis and management of coagulopathy in COVID-19. Further trials on anticoagulation doses in patients with COVID-19 are warranted.

\section{Declarations}

Funding No external funding was used in the preparation of this manuscript.

Conflict of interest Namrata Singhania, Saurabh Bansal, Divya P. Nimmatoori, Abutaleb A. Ejaz, Peter A. McCullough, and Girish Singhania declare they have no potential conflicts of interest that might be relevant to the contents of this manuscript.

Ethics approval Not applicable.

Consent to participate Not applicable.

Consent for publication Not applicable.

Availability of data and materials Not applicable.

Code availability Not applicable.

Author contributions NS wrote the manuscript and reviewed the literature. SB, DPN, AAE, PAM, and GS reviewed the literature and critically revised the manuscript. All authors had access to the data and had a role in writing and reviewing the manuscript.

\section{References}

1. Guan W, Ni Z, Hu Y, Liang W, Ou C, He J, et al. Clinical characteristics of coronavirus disease 2019 in China. N Engl J Med. 2020;382:1708-20. https://doi.org/10.1056/NEJMoa2002032.

2. Singhania N, Bansal S, Singhania G. An atypical presentation of novel coronavirus disease 2019 (COVID-19). Am J Med. 2020;133(7):e365-e366366. https://doi.org/10.1016/j.amjme d.2020.03.026.

3. COVID-19 and coagulopathy: frequently asked questions. https ://www.hematology.org/covid-19/covid-19-and-coagulopathy. Accessed 29 May 2020.

4. Varga Z, Flammer AJ, Steiger P, Haberecker M, Andermatt R, Zinkernagel AS, et al. Endothelial cell infection and endotheliitis in COVID-19. Lancet. 2020;395:1417-8. https://doi.org/10.1016/ S0140-6736(20)30937-5.

5. Ackermann M, Verleden SE, Kuehnel M, Haverich A, Welte T, Laenger F, et al. Pulmonary vascular endothelialitis, thrombosis, 
and angiogenesis in Covid-19. N Engl J Med. 2020;383:120-8. https://doi.org/10.1056/NEJMoa2015432.

6. Begbie M, Notley C, Tinlin S, Sawyer L, Lillicrap D. The factor VIII acute phase response requires the participation of $\mathrm{NF \kappa B}$ and C/EBP. Thromb Haemost. 2000;84:216-22. https://doi. org/10.1055/s-0037-1613999.

7. Magro C, Mulvey JJ, Berlin D, Nuovo G, Salvatore S, Harp J, et al. Complement associated microvascular injury and thrombosis in the pathogenesis of severe COVID-19 infection: a report of five cases. Transl Res. 2020;220:1-13. https://doi.org/10.1016/j. trsl.2020.04.007.

8. Ranucci M, Ballotta A, Di Dedda U, Bayshnikova E, Dei Poli M, Resta M, et al. The procoagulant pattern of patients with COVID-19 acute respiratory distress syndrome. J Thromb Haemost. 2020;18(7):1747-51. https://doi.org/10.1111/jth.14854.

9. Tang N, Bai H, Chen X, Gong J, Li D, Sun Z. Anticoagulant treatment is associated with decreased mortality in severe coronavirus disease 2019 patients with coagulopathy. J Thromb Haemost. 2020;18(5):1094-9. https://doi.org/10.1111/jth.14817.

10. Fox SE, Akmatbekov A, Harbert JL, Li GM, Quincy Brown J, Vander Heide RS. Pulmonary and cardiac pathology in African American patients with COVID-19: an autopsy series from New Orleans. Lancet Respir. 2020;8(7):681-6. https://doi.org/10.1016/ S2213-2600(20)30243-5.

11. Menter T, Haslbauer JD, Nienhold R, Savic S, Hopfer H, Deigendesch N, et al. Post-mortem examination of COVID19 patients reveals diffuse alveolar damage with severe capillary congestion and variegated findings of lungs and other organs suggesting vascular dysfunction. Histopathology. 2020. https://doi.org/10.1111/ his. 14134.

12. Panigada M, Bottino N, Tagliabue P, Grasselli G, Novembrino C, Chantarangkul V, et al. Hypercoagulability of COVID-19 patients in Intensive Care Unit. A report of thromboelastography findings and other parameters of hemostasis. J Thromb Haemost. 2020;18(7):1738-42. https://doi.org/10.1111/jth.14850.

13. Tang N, Li D, Wang X, Sun Z. Abnormal coagulation parameters are associated with poor prognosis in patients with novel coronavirus pneumonia. J Thromb Haemost. 2020;18:844-7. https://doi. org/10.1111/jth. 14768 .

14. Levi M, Toh CH, Thachil J, Watson HG. Guidelines for the diagnosis and management of disseminated intravascular coagulation. Br J Haematol. 2009;145:24-33. https://doi.org/10.111 1/j.1365-2141.2009.07600.x.

15. Wichmann D, Sperhake J-P, Lütgehetmann M, Steurer S, Edler C, Heinemann A, et al. Autopsy findings and venous thromboembolism in patients with COVID-19. Ann Intern Med. 2020. https:// doi.org/10.7326/M20-2003

16. Klok FA, Kruip MJHA, van der Meer NJM, Arbous MS, Gommers DAMPJ, Kant KM, et al. Incidence of thrombotic complications in critically ill ICU patients with COVID-19. Thromb Res. 2020;191:145-7. https://doi.org/10.1016/j.thromres.2020.04.013.

17. Helms J, Tacquard C, Severac F, Leonard-Lorant I, Ohana M, Delabranche X, et al. High risk of thrombosis in patients with severe SARS-CoV-2 infection: a multicenter prospective cohort study. Intensive Care Med. 2020;46(6):1089-98. https://doi. org/10.1007/s00134-020-06062-x.

18. Poissy J, Goutay J, Caplan M, Parmentier E, Duburcq T, Lassalle $\mathrm{F}$, et al. Pulmonary embolism in COVID-19 patients: awareness of an increased prevalence. Circulation. 2020;142(2):184-6. https ://doi.org/10.1161/circulationaha.120.047430.

19. Middeldorp S, Coppens M, van Haaps TF, Foppen M, Vlaar AP, Müller MCA, et al. Incidence of venous thromboembolism in hospitalized patients with COVID-19. J Thromb Haemost. 2020. https ://doi.org/10.1111/jth.14888.

20. Llitjos JF, Leclerc M, Chochois C, Monsallier JM, Ramakers M, Auvray M, et al. High incidence of venous thromboembolic events in anticoagulated severe COVID-19 patients. J Thromb Haemost. 2020;18(7):1743-6. https://doi.org/10.1111/jth.14869.

21. Lodigiani C, Iapichino G, Carenzo L, Cecconi M, Ferrazzi P, Sebastian T, et al. Venous and arterial thromboembolic complications in COVID-19 patients admitted to an academic hospital in Milan, Italy. Thromb Res. 2020;191:9-14. https://doi. org/10.1016/j.thromres.2020.04.024.

22. Artifoni M, Danic G, Gautier G, Gicquel P, Boutoille D, Raffi $\mathrm{F}$, et al. Systematic assessment of venous thromboembolism in COVID-19 patients receiving thromboprophylaxis: incidence and role of D-dimer as predictive factors. J Thromb Thrombolysis. 2020;50(1):211-6. https://doi.org/10.1007/s11239-020-02146-Z.

23. Oxley TJ, Mocco J, Majidi S, Kellner CP, Shoirah H, Singh IP, et al. Large-vessel stroke as a presenting feature of Covid-19 in the young. N Engl J Med. 2020;382:e60. https://doi.org/10.1056/ NEJMc2009787.

24. Bellosta R, Luzzani L, Natalini G, Pegorer MA, Attisani L, Cossu LG, et al. Acute limb ischemia in patients with COVID19 pneumonia. J Vasc Surg. 2020. https://doi.org/10.1016/j. jvs.2020.04.483.

25. Perini P, Nabulsi B, Massoni CB, Azzarone M, Freyrie A. Acute limb ischaemia in two young, non-atherosclerotic patients with COVID-19. Lancet. 2020;395:1546. https://doi.org/10.1016/ S0140-6736(20)31051-5.

26. Stefanini GG, Montorfano M, Trabattoni D, Andreini D, Ferrante $\mathrm{G}$, Ancona M, et al. ST-elevation myocardial infarction in patients with COVID-19: clinical and angiographic outcomes. Circulation. 2020;141(25):2113-6. https://doi.org/10.1161/circulatio naha.120.047525.

27. Cui S, Chen S, Li X, Liu S, Wang F. Prevalence of venous thromboembolism in patients with severe novel coronavirus pneumonia. J Thromb Haemost. 2020;18(6):1421-4. https://doi.org/10.1111/ jth. 14830 .

28. Yao Y, Cao J, Wang Q, Shi Q, Liu K, Luo Z, et al. D-dimer as a biomarker for disease severity and mortality in COVID-19 patients: a case control study. J Intensive Care. 2020;8:49. https ://doi.org/10.1186/s40560-020-00466-z.

29. Yu B, Li X, Chen J, Ouyang M, Zhang H, Zhao X, et al. Evaluation of variation in D-dimer levels among COVID-19 and bacterial pneumonia: a retrospective analysis. J Thromb Thrombolysis. 2020. https://doi.org/10.1007/s11239-020-02171-y.

30. Moores LK, Tritschler T, Brosnahan S, Carrier M, Collen JF, Doerschug K, et al. Prevention, diagnosis, and treatment of VTE in patients with COVID-19. Chest. 2020. https://doi.org/10.1016/j. chest.2020.05.559.

31. Clinical Reference Group SBAR: Therapies for COVID-19. https ://www.bccdc.ca/Health-Professionals-Site/Documents/Guide lines_Unproven_Therapies_COVID-19.pdf. Accessed 17 Jul 2020.

32. Spyropoulos AC, Levy JH, Ageno W, Connors JM, Hunt BJ, Iba T, et al. Scientific and Standardization Committee Communication: clinical guidance on the diagnosis, prevention and treatment of venous thromboembolism in hospitalized patients with COVID19. J Thromb Haemost. 2020. https://doi.org/10.1111/jth.14929.

33. British Thoracic Society issues guidance on VTE in patients with COVID-19. https://brit-thoracic.org.uk/document-library/quali ty-improvement/covid-19/bts-guidance-on-venous-thromboemb olic-disease-in-patients-with-covid-19/. Accessed 17 Jul 2020.

34. Thachil J, Tang N, Gando S, Falanga A, Cattaneo M, Levi M, et al. ISTH interim guidance on recognition and management of coagulopathy in COVID-19. J Thromb Haemost. 2020;18:1023-6. https://doi.org/10.1111/jth.14810.

35. Paranjpe I, Fuster V, Lala A, Russak A, Glicksberg BS, Levin MA, et al. Association of treatment dose anticoagulation with in-hospital survival among hospitalized patients with COVID-19. 
J Am Coll Cardiol. 2020;76(1):122-4. https://doi.org/10.1016/j. jacc.2020.05.001.

36. COVID-19 and VTE/anticoagulation: frequently asked questions. https://www.hematology.org/covid-19/covid-19-and-vte-antic oagulation. Accessed 29 May 2020.
37. Nahum J, Morichau-Beauchant T, Daviaud F, Echegut P, Fichet J, Maillet J-M, et al. Venous thrombosis among critically ill patients with coronavirus disease 2019 (COVID-19). JAMA Netw Open. 2020;3:e2010478. https://doi.org/10.1001/jamanetworkopen .2020 .10478 . 